\title{
Height growth of solutions and a discrete Painlevé equation
}

\author{
A Al-Ghassani1 and R G Halburd?
}

\begin{abstract}
Consider the discrete equation

$$
y_{n+1}+y_{n-1}=\frac{a_{n}+b_{n} y_{n}+c_{n} y_{n}^{2}}{1-y_{n}^{2}}
$$

where the right side is of degree two in $y_{n}$ and where the coefficients $a_{n}, b_{n}$ and $c_{n}$ are rational functions of $n$ with rational coefficients. Suppose that there is a solution such that for all sufficiently large $n$, $y_{n} \in \mathbb{Q}$ and the height of $y_{n}$ dominates the height of the coefficient functions $a_{n}, b_{n}$ and $c_{n}$. We show that if the logarithmic height of $y_{n}$ grows no faster than a power of $n$ then either the equation is a well known discrete Painlevé equation $\mathrm{dP}_{\mathrm{II}}$ or its autonomous version or $y_{n}$ is also an admissible solution of a discrete Riccati equation. This provides further evidence that slow height growth is a good detector of integrability.
\end{abstract}

\section{Introduction}

For discrete equations, integrability appears to be closely related to the slow growth of various measures of complexity [20]. For example, algebraic entropy [6, 14, 5] measures the degree growth of iterates as a function of the initial conditions, while in the Nevanlinna approach [2, 12], one considers the order of growth of meromorphic solutions. A discrete equation on a number field is said to be Diophantine integrable if the logarithmic height of solutions grows polynomially [10]. The last two approaches are connected by Vojta's

\footnotetext{
${ }^{1}$ Department of Mathematics and Statistics, College of Science, Sultan Qaboos University, Al-Khodh P.O. Box 36, P.C 123, Sultanate of Oman

${ }^{2}$ Department of Mathematics, University College London, Gower Street, London WC1E $6 \mathrm{BT}, \mathrm{UK}$
} 
dictionary [21, which relates ideas from Nevanlinna theory (the value distribution of meromorphic functions) to those in Diophantine approximation. See [9] for a survey of different approaches to detecting integrability in discrete systems.

This paper concerns a Diophantine analogue of a classification result of Halburd and Korhonen [11] using Nevanlinna theory. Specifically, we will study discrete equations of the form

$$
y_{n+1}+y_{n-1}=\frac{a_{n}+b_{n} y_{n}+c_{n} y_{n}^{2}}{1-y_{n}^{2}},
$$

where $a_{n}, b_{n}$ and $c_{n}$ are in $\mathbb{Q}(n)$ and the degree of the right side of equation (1) is two. The logarithmic height of a rational number $x=a / b$, where $a$ and $b$ have no common factors, is $h(x)=\log H(x)$, where $H(x)=\max \{|a|,|b|\}$ is the height. A discrete equation such as (11) is said to be Diophantine integrable if the logarithmic height of its solution $y_{n}$ over a number field grows no faster than a power of $n$ [10]. Abarenkova et al [1] used height growth to estimate the entropy of a map. Slow height growth has been used as an efficient numerical test in [15, 4, 17, 16, 7].

The purpose of this paper is to prove the following.

Theorem 1 Let $r_{0}$ be sufficiently large and let $\left(y_{n}\right)_{n \geq r_{0}} \subset \mathbb{Q} \backslash\{-1,1\}$ be a solution of (11), where $a_{n}, b_{n}$ and $c_{n}$ are rational functions of $n$ with coefficients in $\mathbb{Q}$ and the right side of (1) is of degree two in $y_{n}$. If

$$
\sum_{n=r_{0}}^{r} \max \left\{1, h\left(a_{n}\right), h\left(b_{n}\right), h\left(c_{n}\right)\right\}=o\left(\sum_{n=r_{0}}^{r} h\left(y_{n}\right)\right)
$$

as $r \rightarrow \infty$, then either

1. $a_{n}=\alpha n+\beta, b_{n}=\gamma, c_{n}=0$ for constants $\alpha, \beta, \gamma$; or

2. $y_{n}$ also solves the discrete Riccati equation

$$
\begin{aligned}
& \qquad y_{n+1}=\frac{1 / 2\left(a_{n}+\theta b_{n}-2 \theta\right)+y_{n}}{1-\theta y_{n}} \text {, where } \theta=-1 \text { or } 1 \text {; or } \\
& \text { 3. } \limsup _{r \rightarrow \infty} \frac{\log \log \sum_{n=r_{0}}^{r} h\left(y_{n}\right)}{\log r} \geq 1 .
\end{aligned}
$$


This result first appeared in the PhD thesis of the first author.

A solution of equation (11) satisfying (2) will be called admissible. Of the three possible outcomes described in Theorem 1, the first says that equation (11) is the discrete Painlevé equation $\mathrm{dP}_{\mathrm{II}}$ (see Nijhoff and Papageorgiou [18]) or its autonomous version $(\alpha=0)$, the second says that $y_{n}$ solves a well known linearisable discrete Riccati equation and the third implies that $h\left(y_{n}\right)$ grows faster than any power of $n$. If equation (11) has more than two oneparameter families of admissible solutions, they cannot both solve discrete Riccati equations of the form described by the second conclusion unless the equation is $\mathrm{dP}_{\mathrm{II}}$. In case 1 with $\alpha=0$, equation (11) can be derived from the addition law on an elliptic curve, for which it is known that the logarithmic height grows quadratically.

The full version of $\mathrm{dP}_{\mathrm{II}}$ allows $a_{n}$ to have the more general form $a_{n}=$ $\alpha n+\beta+\delta(-1)^{n}$, where $\delta$ is another constant. We do not capture this form as we have assumed that the coefficients $a_{n}, b_{n}$ and $c_{n}$ are rational functions of $n$. This assumption simplifies some of the arguments.

Diophantine integrability is a property of all solutions, not just those that are admissible. Our method involves working with one solution at a time, so an admissibility-type condition is necessary to avoid counterexamples in which $a_{n}, b_{n}$ and $y_{n}$ are chosen arbitrarily and then $c_{n}$ is determined by equation (11).

Of central importance in our proof of Theorem 1 is the fact that there is a simple relationship between the height of a rational number $x$ and a certain sum over all non-trivial absolute values of $x$. For a fixed prime $p$, the $p$-adic absolute value of a non-zero rational number $x$ is $|x|_{p}=p^{-r}$, where $x=\frac{m}{n} p^{r}$ for integers $m, n$ and $r$ such that $p \nmid m n$. The $p$-adic absolute values are non-Archimedean, which means that they satisfy the stronger triangle inequality $|x+y|_{p} \leq \max \left\{|x|_{p},|y|_{p}\right\}$. The usual absolute value, denoted by $|\cdot|_{\infty}$, is Archimedean. Ostrovski's Theorem says that, up to equivalence, the only non-trivial absolute values on $\mathbb{Q}$ are the $p$-adic absolute values, $|\cdot|_{p}$ and the usual absolute value $|\cdot|_{\infty}$. In terms of these absolute values, we have the important identity

$$
h(x)=\sum_{p \leq \infty} \log ^{+}|x|_{p},
$$

where the sum is taken over all finite primes and $p=\infty$ (the "prime at infinity") and $\log ^{+} y:=\max \{0, \log y\}$. 
One of the first properties of discrete equations to be used to identify discrete Painlevé equations was singularity confinement [8, 19], which involves the behaviour of solutions as one iterates through a singularity of the equation. For equation (11), one needs to examine the singular values $y=1$ and $y=-1$. In order to resolve indeterminacies that arise in future iterates, we consider the initial conditions $y_{k-1}=\kappa, y_{k}=\theta+\epsilon$, where $\kappa$ is arbitrary, $\theta^{2}=1$ and $\epsilon$ is small (as we will take the limit $\epsilon \rightarrow 0$ after a finite number of steps). Generically we find that, after taking the limit $\epsilon \rightarrow 0, y_{m}=\infty$ for infinitely many $m>k$. However, for certain choices of $a_{n}, b_{n}$ and $c_{n}$, the singularity appears to be confined to a finite number of iterates.

At the heart of the proof of Theorem 1 are some calculations that look very much like those described above for singularity confinement. The main difference is that we have to consider not only the case in which $1-\theta y_{k}$ is small with respect to the usual absolute value, but also cases in which it is small with respect to $p$-adic absolute values. The identity (41) is eventually used to convert certain statements about absolute values into statements about logarithmic heights. The following theorem is an example of such an expression of singularity (non-)confinement in terms of absolute values. It should be stressed, however, that we do not make assumptions about the long term behaviour of solutions or whether they are eventually confined. For each absolute value $|\cdot|_{p}, \epsilon_{k} \equiv \epsilon_{k, p}$, which is defined precisely in equation (16), determines a length scale in terms of the coefficients $a_{k}, b_{k}, c_{k}$ and a finite number of their shifts.

Theorem 2 Let $\left(y_{n}\right)_{n=k-1}^{k+3} \subset \mathbb{Q} \backslash\{-1,1\}$ satisfy

$$
y_{n+1}+y_{n-1}=\frac{a_{n}+b_{n} y_{n}}{1-y_{n}^{2}},
$$

where $k$ is sufficiently large and the right hand side of the equation is irreducible. Assume that for a fixed absolute value $|\cdot|_{p}(p \leq \infty)$ we have $\left|y_{k-1}\right|_{p} \leq\left|1-\theta y_{k}\right|_{p}^{-1 / 2}$ for $\theta=1$ or -1 . Furthermore, for sufficiently small $\delta>0$, if $\left|1-\theta y_{k}\right|_{p}<\epsilon_{k}$ (where $\epsilon_{k}$ is defined in (16)), then

$$
\begin{aligned}
& \text { (i) } y_{k+1}=\frac{a_{k}+\theta b_{k}}{2\left(1-\theta y_{k}\right)}+A_{k} \text {, where }\left|A_{k}\right|_{p} \leq\left|1-\theta y_{k}\right|_{p}^{-1 / 2} \text { if } p<\infty \text { and if } \\
& \quad p=\infty,\left|A_{k}\right|_{\infty} \leq \frac{11}{10} \cdot\left|1-\theta y_{k}\right|_{\infty}^{-1 / 2} \text {. } \\
& \text { (ii) } y_{k+2}=-\theta+\left(\frac{\theta a_{k}+b_{k}-2 b_{k+1}}{a_{k}+\theta b_{k}}\right)\left(1-\theta y_{k}\right)+B_{k},
\end{aligned}
$$




$$
\begin{aligned}
& \text { where }\left|B_{k}\right|_{p} \leq\left|1-\theta y_{k}\right|_{p}^{3 / 2-5 \delta} \text { if } p<\infty \text { and if } p=\infty,\left|B_{k}\right|_{\infty} \leq \frac{1}{2} \cdot \mid 1- \\
& \left.\theta y_{k}\right|_{\infty} ^{3 / 2-5 \delta} . \\
& \text { (iii) } y_{k+3}=\frac{\left(a_{k+2}-\theta b_{k+2}-\theta\left(\theta a_{k}+b_{k}-2 b_{k+1}\right)\right)}{2\left(1+\theta y_{k+2}\right)}+C_{k} \text {, where }\left|C_{k}\right|_{p} \leq \mid 1+ \\
& \left.\theta y_{k+2}\right|_{p} ^{-(2 / 3+2 \delta)} \text { if } p<\infty \text { and if } p=\infty,\left|C_{k}\right|_{\infty} \leq 2\left|1+\theta y_{k+2}\right|_{\infty}^{-(2 / 3+2 \delta)} .
\end{aligned}
$$

Theorems 1 and 2 can easily be extended to arbitrary number fields (finite field extensions of the rationals) as there is a simple analogue of the identity (4) in this case.

In [10], it was shown that if an equation of the form

$$
y_{n+1}+y_{n-1}=R\left(n, y_{n}\right)
$$

where $R$ is rational, has an admissible solution, then $\operatorname{deg}_{y} R(n, y) \leq 2$. The case $R\left(n, y_{n}\right)=\left(a_{n}+b_{n} y_{n}+c_{n} y_{n}^{2}\right) / y_{n}^{2}$ was studied in [13. There are essential technical difficulties which distinguish the two cases and consequently the analysis used to treat each of them. The fact that, for certain $a_{n}, b_{n}$ and $c_{n}$, there are solutions of equation (11) that also satisfy discrete Riccati equations requires a more subtle analysis.

\section{Proof of Theorem 1}

First we consider the case in which $c_{n} \not \equiv-2,0$ or 2 . We introduce a quantity $\epsilon_{n}$, which provides a scale with respect to which we measure distances between iterates and certain singular values. For any finite set of rational functions $\left\{f_{1}, \ldots, f_{m}\right\}$ of $n$, there exists $K \in \mathbb{N}$ such that for every function $f_{j}$ that is not identically zero, $f_{j}(n)$ is finite and nonzero for all $n \geq K$. Throughout this paper we will refer to such an integer $K$, which may need to be increased a finite number of times, without further comment. Since the right side of equation (1) is of degree 2, neither of the rational functions $a_{n}+b_{n}+c_{n}$ nor $a_{n}-b_{n}+c_{n}$ vanishes identically. For $n>K$, define the sets $X_{n, 0}=\left\{1 / 2, b_{n}, c_{n}, c_{n-1}^{-1}, c_{n+1}^{-1}\right\}, X_{n, \pm}=\left\{\left(a_{n} \pm b_{n}+c_{n}\right)^{-1},\left(a_{n+1} \pm\right.\right.$ $\left.\left.b_{n+1}+c_{n+1}\right) / 2,\left(a_{n-1} \pm b_{n-1}+c_{n-1}\right) / 2,\left(c_{n+1} \pm 2\right)^{-1},\left(c_{n-1} \pm 2\right)^{-1}\right\}$ and let $X_{n}=X_{n,+} \cup X_{n, 0} \cup X_{n,-}$. For a fixed sufficiently small $\delta>0$ we define $\epsilon_{n}$ $(\forall n>K)$ by

$$
\epsilon_{n}^{-\delta}=\kappa_{p} \max _{x \in X_{n}}\left\{|x|_{p}\right\}
$$


where $\kappa_{p}=1$ for $p<\infty$ and $\kappa_{\infty}=10$. Equation (6) allows us to estimate certain combinations of the coefficients $a_{n}, b_{n}, c_{n}$ in terms of $\epsilon_{n}$. For example, for $p<\infty$, we have $\left|c_{n}\right|_{p} \leq \epsilon_{n}^{-\delta} \Rightarrow \epsilon_{n}^{\delta} \leq\left|c_{n}\right|_{p}^{-1}$. For the Archimedean absolute value $(p=\infty)$, we have $10\left|c_{n}\right|_{\infty} \leq \epsilon_{n}^{-\delta} \Rightarrow \epsilon_{n}^{\delta} \leq \frac{1}{10}\left|c_{n}\right|_{\infty}^{-1}$.

Lemma 3 Let $\left(y_{n}\right) \subset \mathbb{Q} \backslash\{-1,1\}$ be a solution of equation (1) where $a_{n}, b_{n}$ and $c_{n}$ are in $\mathbb{Q}(n)$ and $c_{n}$ is a rational function not identically 0 or \pm 2 . Furthermore, assume that the numerator and the denominator of (11) are coprime. For a fixed prime $p \leq \infty$ and $k>K$, let $\epsilon_{k}$ be as defined in (6) $)$. If $\left|1-\theta y_{k}\right|_{p}<\epsilon_{k}$ for $\theta=1$ or -1 , then either

$$
\left|y_{k+1}\right|_{p} \geq \frac{1}{\left|1-\theta y_{k}\right|_{p}^{1-\delta}} \text { and } \quad\left|1 \pm \theta y_{k+2}\right|_{p} \geq \epsilon_{k+2}
$$

or

$$
\left|y_{k-1}\right|_{p} \geq \frac{1}{\left|1-\theta y_{k}\right|_{p}^{1-\delta}} \text { and }\left|1 \pm \theta y_{k-2}\right|_{p} \geq \epsilon_{k-2}
$$

Proof : The definition of $\epsilon_{n}^{-\delta}$ in (6) implies that $\epsilon_{n} \leq 1$. Furthermore when $p=\infty$ or $p=2, \epsilon_{n}<1$. First we consider the non-Archimedean case for a fixed prime $p<\infty$. Let $\left|1-\theta y_{k}\right|_{p}<\epsilon_{k}$ for some $k>K$, where $\theta=1$ or -1 . From equation (11) we have

$$
\left(y_{k+1}+y_{k-1}\right)\left(1+\theta y_{k}\right)=\frac{a_{k}+\theta b_{k}+c_{k}}{1-\theta y_{k}}-\theta b_{k}-c_{k}\left(1+\theta y_{k}\right) .
$$

So from equations (6) and (7),

$$
\begin{gathered}
\left|1-\theta y_{k}\right|_{p}^{-(1-\delta)}<\frac{\epsilon_{k}^{\delta}}{\left|1-\theta y_{k}\right|_{p}} \leq \frac{\left|a_{k}+\theta b_{k}+c_{k}\right|_{p}}{\left|1-\theta y_{k}\right|_{p}} \\
\leq \quad\left|\left(y_{k+1}+y_{k-1}\right)\left(1+\theta y_{k}\right)+\theta b_{k}+c_{k}\left(1+\theta y_{k}\right)\right|_{p} \\
\leq \quad \max \left\{\left|y_{k+1}+y_{k-1}\right|_{p} \cdot\left|1+\theta y_{k}\right|_{p},\left|b_{k}\right|_{p},\left|c_{k}\right|_{p} \cdot\left|1+\theta y_{k}\right|_{p}\right\} .
\end{gathered}
$$

From (16),$\left|b_{k}\right|_{p} \leq \epsilon_{k}^{-\delta} \leq \epsilon_{k}^{-(1-\delta)}<\left|1-\theta y_{k}\right|_{p}^{-(1-\delta)}$. Similarly, $\left|c_{k}\right|_{p}<\mid 1-$ $\left.\theta y_{k}\right|_{p} ^{-(1-\delta)}$. Also, $\left|1+\theta y_{k}\right|_{p}=\left|2-\left(1-\theta y_{k}\right)\right|_{p} \leq \max \left\{|2|_{p},\left|1-\theta y_{k}\right|_{p}\right\} \leq$ $\max \left\{1, \epsilon_{k}\right\}=1$. Therefore, (8) reduces to $\left|1-\theta y_{k}\right|_{p}^{-(1-\delta)} \leq\left|y_{k+1}+y_{k-1}\right|_{p} \leq$ $\max \left\{\left|y_{k+1}\right|_{p},\left|y_{k-1}\right|_{p}\right\}$. Without loss of generality, we choose the maximum to be $\left|y_{k+1}\right|_{p}$ and for the rest of the proof we use $\left|y_{k+1}\right|_{p} \geq\left|1-\theta y_{k}\right|_{p}^{-(1-\delta)}$. 
From $\left|1-\theta y_{k}\right|_{p}<\epsilon_{k}$ and $\left|y_{k+1}\right|_{p} \geq\left|1-\theta y_{k}\right|_{p}^{-(1-\delta)}$, we have

$$
\epsilon_{k}^{-(1-\delta)}<\left|y_{k+1}\right|_{p} \leq \max \left\{1,\left|1 \pm y_{k+1}\right|_{p}\right\}=\left|1 \pm y_{k+1}\right|_{p} .
$$

Rewriting equation (1) as $y_{k+2}+c_{k+1}+\theta=\left(a_{k+1}+b_{k+1}+c_{k+1}\right) /\left[2\left(1-y_{k+1}\right)\right]+$ $\left(a_{k+1}-b_{k+1}+c_{k+1}\right) /\left[2\left(1+y_{k+1}\right)\right]+\theta\left(1-\theta y_{k}\right)$, we have $\left|y_{k+2}+c_{k+1}+\theta\right|_{p}<\epsilon_{k}^{1-2 \delta}$. From (므),

$$
\begin{aligned}
\epsilon_{k} & \leq \epsilon_{k}^{\delta} \leq\left|c_{k+1}+\theta \pm \theta\right|_{p}=\left|\left(y_{k+2}+c_{k+1}+\theta\right) \pm \theta\left(1 \mp \theta y_{k+2}\right)\right|_{p} \\
& \leq \max \left\{\left|y_{k+2}+c_{k+1}+\theta\right|_{p},\left|1 \mp \theta y_{k+2}\right|_{p}\right\}=\left|1 \mp \theta y_{k+2}\right|_{p},
\end{aligned}
$$

for $\delta<1 / 3$, which proves the lemma for non-Archimedean absolute values $(p<\infty)$.

The Archimedean case $(p=\infty)$ is similar. We have

$$
\begin{aligned}
& 10\left|1-\theta y_{k}\right|_{\infty}^{-(1-\delta)}<\frac{10 \epsilon_{k}^{\delta}}{\left|1-\theta y_{k}\right|_{\infty}} \leq \frac{\left|a_{k}+\theta b_{k}+c_{k}\right|_{\infty}}{\left|1-\theta y_{k}\right|_{\infty}} \\
\leq & \left|\left(y_{k+1}+y_{k-1}\right)\left(1+\theta y_{k}\right)+\theta b_{k}+c_{k}\left(1+\theta y_{k}\right)\right|_{\infty} \\
\leq & \left|y_{k+1}+y_{k-1}\right|_{\infty} \cdot\left|1+\theta y_{k}\right|_{\infty}+\left|b_{k}\right|_{\infty}+\left|c_{k}\right|_{\infty} \cdot\left|1+\theta y_{k}\right|_{\infty} .
\end{aligned}
$$

Also $\left|1+\theta y_{k}\right|_{\infty} \leq\left|1-\theta y_{k}\right|_{\infty}+|2|_{\infty}<\epsilon_{k}+2<3$. Finally we have from (16) that $\left|b_{k}\right|_{\infty}<\left|1-\theta y_{k}\right|_{\infty}^{-(1-\delta)}$ and $\left|c_{k}\right|_{\infty}<\left|1-\theta y_{k}\right|_{\infty}^{-(1-\delta)}$. So (9) gives $10\left|1-\theta y_{k}\right|_{\infty}^{-(1-\delta)}<3\left|y_{k+1}+y_{k-1}\right|_{\infty}+\left|b_{k}\right|_{\infty}+3\left|c_{k}\right|_{\infty}<3\left|y_{k+1}+y_{k-1}\right|_{\infty}+4 \mid 1-$ $\left.\theta y_{k}\right|_{\infty} ^{-(1-\delta)}$. Therefore, $2\left|1-\theta y_{k}\right|_{\infty}^{-(1-\delta)} \leq\left|y_{k+1}+y_{k-1}\right|_{\infty} \leq\left|y_{k+1}\right|_{\infty}+\left|y_{k-1}\right|_{\infty}$. Hence, either $\left|y_{k+1}\right|_{\infty} \geq\left|1-\theta y_{k}\right|_{\infty}^{-(1-\bar{\delta})}$ or $\left|y_{k-1}\right|_{\infty} \geq\left|1-\theta y_{k}\right|_{\infty}^{-(1-\delta)}$, which proves the first assertion of the lemma. Without loss of generality, we take $\left|y_{k+1}\right|_{\infty} \geq\left|1-\theta y_{k}\right|_{\infty}^{-(1-\delta)}$.

We have $\left|1 \pm y_{k+1}\right|_{\infty}^{-1}<\frac{5}{4} \epsilon_{k}^{(1-\delta)}$, so $\left|y_{k+2}+c_{k+1}+\theta\right|_{\infty} \leq \mid a_{k+1}+b_{k+1}+$ $\left.c_{k+1}\right|_{\infty} /\left(2\left|1-y_{k+1}\right|_{\infty}\right)+\left|a_{k+1}-b_{k+1}+c_{k+1}\right|_{\infty} /\left(2\left|1+y_{k+1}\right|_{\infty}\right)+\left|1-\theta y_{k}\right|_{\infty}<$ $\frac{1}{8} \epsilon_{k}^{1-2 \delta}+\frac{1}{8} \epsilon_{k}^{1-2 \delta}+\epsilon_{k}<9 \epsilon_{k}^{\delta}$. This gives $10 \epsilon_{k}^{\delta} \leq\left|c_{k+1}+\theta \pm \theta\right|_{\infty}=\mid\left(y_{k+2}+c_{k+1}+\right.$ $\theta) \pm\left.\theta\left(1 \mp \theta y_{k+2}\right)\right|_{\infty}<9 \epsilon_{k}^{\delta}+\left|1 \mp \theta y_{k+2}\right|_{\infty}$. Hence, $\left|y_{k+2}+c_{k+1}+\theta\right|_{\infty} \leq 9 \epsilon_{k}^{\delta}<$ $9\left|1 \mp \theta y_{k+2}\right|_{\infty}$. Now $10 \epsilon_{k+2}<10 \epsilon_{k+2}^{\delta} \leq\left|c_{k+1}+\theta \pm \theta\right|_{\infty}=\mid\left(y_{k+2}+c_{k+1}+\theta\right) \pm$ $\left.\theta\left(1 \mp \theta y_{k+2}\right)\right|_{\infty} \leq 10\left|1 \mp \theta y_{k+2}\right|_{\infty}$.

We are now ready to prove the following.

Theorem 4 Let $\left(y_{n}\right) \subset \mathbb{Q} \backslash\{-1,1\}$ be an admissible solution of the equation (11), where $a_{n}, b_{n}$ and $c_{n}$ are rational functions of $n$ with $c_{n} \neq 0$ or \pm 2 and the right hand side of (11) is of degree 2 in $y_{n}$ for all sufficiently large $n$. Then 
there exists an integer $r_{0}$ such that for all $r \geq r_{0}$ and $F<2$, the summed logarithmic height

$$
h_{r}\left(y_{n}\right)=\sum_{n=r_{0}}^{r} h\left(y_{n}\right)=\sum_{n=r_{0}}^{r} \sum_{p \leq \infty} \log ^{+}\left|y_{n}\right|_{p},
$$

satisfies $h_{r}\left(y_{n}\right) \geq F^{r} D$ for some $D>0$.

Proof : We will show that there is a number $\tau<2$ such that for each absolute value $|\cdot|_{p}(\forall p \leq \infty)$ on $\mathbb{Q}$ and for all $r \geq r_{0}$,

$$
\sum_{n=r_{0}}^{r}\left(\log ^{+} \frac{1}{\left|1-y_{n}\right|_{p}}+\log ^{+} \frac{1}{\left|1+y_{n}\right|_{p}}\right) \leq \tau \sum_{n=r_{0}}^{r+1} \log ^{+}\left|y_{n}\right|_{p} .
$$

We can then sum this inequality over all absolute values to show that the summed logarithmic height grows exponentially.

Fix a prime $p \leq \infty$ and an integer $r_{0}>K$ and define the four sets

$$
\begin{aligned}
& A_{r}^{ \pm}=\left\{n: r_{0} \leq n \leq r \text { and }\left|1 \mp y_{n}\right|_{p}<\epsilon_{n}\right\}, \\
& B_{r}^{ \pm}=\left\{n: r_{0} \leq n \leq r \text { and } \epsilon_{n} \leq\left|1 \mp y_{n}\right|_{p}<1\right\},
\end{aligned}
$$

where $\epsilon_{n}$ is given by equation ([6) . We now show that $A_{r}^{+} \cap A_{r}^{-}=\emptyset$. For any $n \in A_{r}^{+}$we have $\left|1-y_{n}\right|<\epsilon_{n}$. If $p<\infty$,

$$
\epsilon_{n} \leq \epsilon_{n}^{\delta} \leq|2|_{p} \leq \max \left\{\left|1-y_{n}\right|_{p},\left|1+y_{n}\right|_{p}\right\}=\left|1+y_{n}\right|_{p},
$$

so $n \notin A_{r}^{-}$. The same conclusion holds in the Archimedean case since $\epsilon_{n}<1$ and so

$$
1<|2|_{\infty}-\epsilon_{n} \leq\left|1-y_{n}\right|_{\infty}+\left|1+y_{n}\right|_{\infty}-\epsilon_{n}<\left|1+y_{n}\right|_{\infty} .
$$

Lemma 3 shows that for each $n \in A_{r}^{ \pm}$, we can define $\sigma_{n}^{ \pm}=-1$ or 1 such that $\left|y_{n+\sigma_{n}^{ \pm}}\right|_{p} \geq \frac{1}{\left|1 \mp y_{n}\right|_{p}^{1-\delta}}$. Lemma 3 also shows that $\left\{n+\sigma_{n}^{+} \mid n \in A_{r}^{+}\right\} \cap\{n+$ $\left.\sigma_{n}^{-} \mid n \in A_{r}^{-}\right\}=\emptyset$ and that

$$
\begin{aligned}
& \sum_{k \in A_{r}^{+}} \log ^{+} \frac{1}{\left|1-y_{k}\right|_{p}}+\sum_{k \in A_{r}^{-}} \log ^{+} \frac{1}{\left|1+y_{k}\right|_{p}} \\
\leq & \frac{1}{1-\delta}\left(\sum_{k \in A_{r}^{+}} \log ^{+}\left|y_{k+\sigma_{k}^{+}}\right|_{p}+\sum_{k \in A_{r}^{-}} \log ^{+}\left|y_{k+\sigma_{k}^{-}}\right|_{p}\right) \\
\leq & \frac{1}{1-\delta} \sum_{k=r_{0}-1}^{r+1} \log ^{+}\left|y_{k}\right|_{p} .
\end{aligned}
$$


Recalling the definition of $\epsilon_{k}$ in (6) , we have

$$
\begin{aligned}
& \sum_{k \in B_{r}^{ \pm}} \log ^{+} \frac{1}{\left|1 \pm y_{k}\right|_{p}} \leq \sum_{k \in B_{r}^{ \pm}} \log ^{+} \epsilon_{k}^{-1}=\frac{1}{\delta} \sum_{k \in B_{r}^{ \pm}} \log ^{+}\left(\kappa_{p} \max _{x \in X_{k}}\left\{|x|_{p}\right\}\right) \\
\leq & \frac{1}{\delta} \sum_{k=r_{0}}^{r}\left(\log ^{+} \kappa_{p}+\sum_{x \in X_{k}} \log ^{+}|x|_{p}\right)=: M_{p}
\end{aligned}
$$

So from the inequalities (11) and (12) we see that for all primes $p \leq \infty$,

$$
\sum_{k=r_{0}}^{r}\left(\log ^{+} \frac{1}{\left|1-y_{k}\right|_{p}}+\log ^{+} \frac{1}{\left|1+y_{k}\right|_{p}}\right) \leq \frac{1}{1-\delta} \sum_{k=r_{0}-1}^{r+1} \log ^{+}\left|y_{k}\right|_{p}+2 M_{p} \text {. }
$$

To get the height, we sum over all the primes $(p \leq \infty)$ which yields

$$
\sum_{k=r_{0}}^{r} h\left(\frac{1}{1-y_{k}}\right)+\sum_{k=r_{0}}^{r} h\left(\frac{1}{1+y_{k}}\right) \leq \frac{1}{1-\delta} \sum_{k=r_{0}-1}^{r+1} h\left(y_{k}\right)+R_{r}
$$

where

$$
R_{r}=\frac{2}{\delta} \sum_{k=r_{0}}^{r} \sum_{x \in X_{k}} h(x)+\frac{r-r_{0}}{2} \log 10=o\left(\sum_{k=r_{0}-1}^{r+1} h\left(y_{k}\right)\right),
$$

where the second equality follows from our admissibility condition (2). Furthermore, we have $\left|h\left(\left(1-\theta y_{k}\right)^{-1}\right)-h\left(y_{k}\right)\right|_{\infty} \leq \log 2$, where $\theta=1$ or -1 . So we see that the summed logarithmic height satisfies $h_{r+1}\left(y_{k}\right) \geq 2(1-$ $\delta) h_{r}\left(y_{k}\right)+o\left(h_{r+1}\left(y_{k}\right)\right)$ and hence for any $\nu>0$ there is a constant $D>0$ such that

$$
h_{r}\left(y_{k}\right) \geq\left(\frac{2(1-\delta)}{1+\nu}\right)^{r} D
$$

For sufficiently small $\delta, \nu, 1<F=\frac{2(1-\delta)}{1+\nu}<2$, which proves the theorem.

Now we consider the case in which $c_{n}$ vanishes identically, i.e.

$$
y_{n+1}+y_{n-1}=\frac{a_{n}+b_{n} y_{n}}{1-y_{n}^{2}} .
$$

Our strategy is again to prove an inequality of the form (10) with $\tau<2$. The integer $K$ is chosen such that for all $n>K, a_{n}+b_{n}$ and $a_{n}-b_{n}$ are 
nonzero and each of the expressions $\pm a_{n}+b_{n}-2 b_{n+1}, \pm a_{n}+b_{n}-2 b_{n-1}$ and $a_{n} \pm b_{n} \pm\left( \pm a_{n-2}+b_{n-2}-2 b_{n-1}\right)$ is either identically zero or for all $n>K$ it is nonzero.

In the following definition of $\epsilon_{n}$ we take the maximum over a set for which we ignore those elements that are undefined (or infinite) and take the maximum of all the remaining finite elements. For sufficiently small $\delta>0$ and for all $n>K$ we define $\epsilon_{n}$ by

$$
\begin{aligned}
& \epsilon_{n}^{-\delta}=\kappa_{p} \max \left\{\quad|2|_{p}^{-1},|1 / 2|_{p} \cdot\left|a_{n} \pm b_{n}\right|_{p},|1 / 2|_{p}^{-1} \cdot\left|a_{n} \pm b_{n}\right|_{p}^{-1},\left|a_{n+1}\right|_{p},\left|b_{n+1}\right|_{p},\right. \\
& \left|a_{n-1}\right|_{p},\left|b_{n-1}\right|_{p},|1 / 2|_{p} \cdot\left|a_{n+2} \pm b_{n+2}\right|_{p},|1 / 2|_{p} \cdot\left|a_{n-2} \pm b_{n-2}\right|_{p}, \\
& \left| \pm a_{n}+b_{n}-2 b_{n+1}\right|_{p},\left| \pm a_{n}+b_{n}-2 b_{n-1}\right|_{p},\left|a_{n} \pm b_{n}\right|_{p}^{-1} \text {, } \\
& \left| \pm a_{n}+b_{n}-2 b_{n+1}\right|_{p}^{-1},\left| \pm a_{n}+b_{n}-2 b_{n-1}\right|_{p}^{-1},\left|a_{n} \pm b_{n}\right|_{p} \text {, } \\
& \left.|1 / 2|_{p}^{-1} \cdot\left|a_{n} \mp b_{n} \mp\left( \pm a_{n-2}+b_{n-2}-2 b_{n-1}\right)\right|_{p}^{-1}\right\} \text {, }
\end{aligned}
$$

where $\kappa_{p}=1$ if $p<\infty$ and $\kappa_{\infty}=10$. It is evident from the definition that $\epsilon_{n} \leq 1$ when $p<\infty$ and $p \neq 2$, while $\epsilon_{n}<1$ when $p=\infty$ or $p=2$.

We again define the sets $A_{r}^{ \pm}$and $B_{r}^{ \pm}$as in (11). The points of $A_{r}^{ \pm}$will be called \pm 1 points (since $y_{n}$ is close to \pm 1 with respect to the absolute value). As in the proof of Theorem 4, it can be shown that if $\left|1-\theta y_{n}\right|_{p}<\epsilon_{n}$ for $\theta=1$ or -1 , then $\left|1+\theta y_{n}\right|_{p} \geq \epsilon_{n}$. Hence $A_{r}^{+} \cap A_{r}^{-}=\emptyset$. The admissibility of $y_{n}$ then implies that

$\sum_{n=r_{0}}^{r}\left(\log ^{+} \frac{1}{\left|1-y_{n}\right|_{p}}+\log ^{+} \frac{1}{\left|1+y_{n}\right|_{p}}\right)=\sum_{n \in A_{r}^{+}} \log ^{+} \frac{1}{\left|1-y_{n}\right|_{p}}+\sum_{n \in A_{r}^{-}} \log ^{+} \frac{1}{\left|1+y_{n}\right|_{p}}+\Phi_{r}$

where $\sum_{p \leq \infty} \Phi_{r}=o\left(h_{r+1}\left(y_{n}\right)\right)$.

We construct a number of disjoint subintervals containing only 1 points, -1 points and points where $y_{n}$ is sufficiently large to make a significant contribution to the right hand side of the inequality (10).

Definition 5 Suppose that $\left|1-\theta y_{k}\right|_{p}<\epsilon_{k}$, for some $k \in \mathbb{Z}$ and $\theta=1$ or $\theta=-1$. Then the oscillating sequence $S$ containing $k$ is the longest interval in $\mathbb{Z}$ (possibly unbounded) satisfying the following conditions.

1. If $k+2 l \in S$ then $\left|1-(-1)^{l} \theta y_{k+2 l}\right|_{p}<\epsilon_{k+2 l}$;

2. If $\{k+2 l-1, k+2 l\} \subseteq S$, then $\left|y_{k+2 l-1}\right|_{p} \geq\left|1-(-1)^{l} \theta y_{k+2 l}\right|_{p}^{-(1-\delta)}$; and 
3. If $\{k+2 l, k+2 l+1\} \subseteq S$, then $\left|y_{k+2 l+1}\right|_{p} \geq\left|1-(-1)^{l} \theta y_{k+2 l}\right|_{p}^{-(1-\delta)}$.

If $\left|1-\theta y_{n}\right|_{p}<\epsilon_{n}$ then either $\left|y_{n+1}\right|_{p} \geq\left|1-\theta y_{n}\right|_{p}^{-(1-\delta)}$ or $\left|y_{n-1}\right|_{p} \geq \mid 1-$ $\left.\theta y_{n}\right|_{p} ^{-(1-\delta)}$, so every \pm 1 point lies in an oscillating sequence containing at least two elements. For a fixed oscillating sequence $S$ and $r \geq r_{0}$, we will now obtain a suitable upper bound for

$$
\sum_{n \in S \cap A_{r}^{+}} \log ^{+} \frac{1}{\left|1-y_{n}\right|_{p}}+\sum_{n \in S \cap A_{r}^{-}} \log ^{+} \frac{1}{\left|1+y_{n}\right|_{p}} .
$$

Case 1: Let $m+1$ be the total number of 1 points and -1 points in $S \cap\left[r_{0}, r\right]$ and assume that $m \geq 2$. Let $I$ be the shortest subinterval of $S \cap\left[r_{0}, r\right]$ containing these \pm 1 points. Let $k$ be the first term in $I$, so that $\left|1-\theta y_{k}\right|_{p}<\epsilon_{k}$ for some choice of $\theta=-1$ or 1 . Then $I=\{k, k+1, \ldots, k+2 m\}$ and contains exactly $m$ points on which $y_{n}$ is big in the sense that $\left|y_{k+1}\right|_{p} \geq \mid 1-$ $\left.\theta y_{k}\right|_{p} ^{-(1-\delta)},\left|y_{k+2 m-1}\right|_{p} \geq\left|1-(-1)^{m} \theta y_{k+2 m}\right|_{p}^{-(1-\delta)}$ and $\left|y_{k+2 l+1}\right|_{p} \geq \max \{\mid 1-$ $\left.\left.(-1)^{l} \theta y_{k+2 l}\right|_{p} ^{-(1-\delta)},\left|1-(-1)^{l+1} \theta y_{k+2 l+2}\right|_{p}^{-(1-\delta)}\right\}$, for all $l=1, \ldots, m-2$. Hence

$$
\begin{array}{r}
\sum_{n \in S \cap A_{r}^{+}} \log ^{+} \frac{1}{\left|1-y_{n}\right|_{p}}+\sum_{n \in S \cap A_{r}^{-}} \log ^{+} \frac{1}{\left|1+y_{n}\right|_{p}} \\
=\sum_{l=0}^{m} \log ^{+} \frac{1}{\left|1-(-1)^{l} \theta y_{k+2 l}\right|_{p}} \\
=\sum_{l=1}^{m} \frac{l}{m} \log ^{+} \frac{1}{\left|1-(-1)^{l} \theta y_{k+2 l}\right|_{p}}+\sum_{l=0}^{m-1} \frac{m-l}{m} \log ^{+} \frac{1}{\left|1-(-1)^{l} \theta y_{k+2 l}\right|_{p}} \\
=\sum_{l=0}^{m-1} \frac{l+1}{m} \log ^{+} \frac{1}{\left|1-(-1)^{l+1} \theta y_{k+2 l+2}\right|_{p}}+\sum_{l=0}^{m-1} \frac{m-l}{m} \log ^{+} \frac{1}{\left|1-(-1)^{l} \theta y_{k+2 l}\right|_{p}} \\
\leq \frac{1}{1-\delta} \sum_{l=0}^{m-1}\left(\frac{l+1}{m}+\frac{m-l}{m}\right) \log ^{+}\left|y_{k+2 l+1}\right|_{p} \\
=\frac{m+1}{(1-\delta) m} \sum_{l=0}^{m-1} \log ^{+}\left|y_{k+2 l+1}\right|_{p}=\frac{m+1}{(1-\delta) m} \sum_{n \in S \cap\left[r_{0}, r\right]} \log ^{+}\left|y_{n}\right|_{p} \\
\leq \frac{3}{2(1-\delta)} \sum_{n \in S \cap\left[r_{0}, r\right]} \log ^{+}\left|y_{n}\right|_{p},
\end{array}
$$


where the last inequality follows from $m \geq 2$.

Case 2: There are exactly two \pm 1 points in $S \cap\left[r_{0}, r\right]$. Define $k$ such that these points are $k$ and $k+2$. That is, for some choice of $\theta= \pm 1$, we have $\left|1-\theta y_{k}\right|_{p}<\epsilon_{k}$ and $\left|1+\theta y_{k+2}\right|_{p}<\epsilon_{k+2}$. We will use the following corollary of Theorem 2 ,

Corollary 6 Fix a prime $p \leq \infty$. For some $k>K$ let $\epsilon_{k}$ be given (16) and suppose that for $\theta=1$ or $-1,\left|1-\theta y_{k}\right|_{p}<\epsilon_{k},\left|y_{k-1}\right|_{p} \leq\left|1-\theta y_{k}\right|_{p}^{-1 / 2}$ and $\left|1+\theta y_{k+2}\right|_{p}<\epsilon_{k+2}$. Assume that $a_{k}-\theta b_{k}-\theta\left(\theta a_{k-2}+b_{k-2}-2 b_{k-1}\right) \not \equiv 0$, then $\left|y_{k+3}\right|_{p}>\left|1+\theta y_{k+2}\right|_{p}^{-1 / 2}$.

Proof: We begin with the non-Archimedean case $p<\infty$. From part (iii) of Theorem 2 and the definition (16) , we have

$$
\begin{aligned}
\left|1+\theta y_{k+2}\right|_{p}^{-(1-\delta)} & <\frac{\epsilon_{k+2}^{\delta}}{\left|1+\theta y_{k+2}\right|_{p}} \leq \frac{\left|a_{k+2}-\theta b_{k+2}-\theta\left(\theta a_{k}+b_{k}-2 b_{k+1}\right)\right|_{p}}{|2|_{p} \cdot\left|1+\theta y_{k+2}\right|_{p}} \\
& =\left|y_{k+3}-C_{k}\right|_{p} \leq \max \left\{\left|y_{k+3}\right|_{p},\left|C_{k}\right|_{p}\right\}
\end{aligned}
$$

From Theorem 2 we have that for sufficiently small $\delta>0,\left|C_{k}\right|_{p} \leq \mid 1+$ $\left.\theta y_{k+2}\right|_{p} ^{-2 / 3-2 \delta} \leq\left|1+\theta y_{k+2}\right|_{p}^{-(1-\delta)}$. So (18) reduces to $\left|y_{k+3}\right|_{p}>\left|1+\theta y_{k+2}\right|_{p}^{-(1-\delta)} \geq$ $\left|1+\theta y_{k+2}\right|_{p}^{-1 / 2}$.

For the Archimedean absolute value, $\kappa_{\infty}=10$ in (16), giving

$$
\begin{aligned}
& 10\left|1+\theta y_{k+2}\right|_{p}^{-(1-\delta)}<\frac{10 \epsilon_{k+2}^{\delta}}{\left|1+\theta y_{k+2}\right|_{p}} \\
\leq & \frac{\left|a_{k+2}-\theta b_{k+2}-\theta\left(\theta a_{k}+b_{k}-2 b_{k+1}\right)\right|_{p}}{|2|_{p} \cdot\left|1+\theta y_{k+2}\right|_{p}}=\left|y_{k+3}-C_{k}\right|_{p} \leq\left|y_{k+3}\right|_{p}+\left|C_{k}\right|_{p} \\
\leq & \left|y_{k+3}\right|_{p}+2\left|1+\theta y_{k+2}\right|_{p}^{-2 / 3-2 \delta} \leq\left|y_{k+3}\right|_{p}+9\left|1+\theta y_{k+2}\right|_{p}^{-(1-\delta)} .
\end{aligned}
$$

So $\left|y_{k+3}\right|_{p}>\left|1+\theta y_{k+2}\right|_{p}^{-(1-\delta)} \geq\left|1+\theta y_{k+2}\right|_{p}^{-1 / 2}$ which completes the proof.

Hence if $a_{k}-\theta b_{k}-\theta\left(\theta a_{k-2}+b_{k-2}-2 b_{k-1}\right) \not \equiv 0$, then either $\left|y_{k-1}\right|_{p}>$ $\left|1-\theta y_{k}\right|_{p}^{-1 / 2}$ or $\left|y_{k+3}\right|_{p}>\left|1+\theta y_{k+2}\right|_{p}^{-1 / 2}$. This says that, even if neither $k-1$ nor $k+3$ is in $S$, at least one of $y_{k-1}$ or $y_{k+3}$ has to be moderately large. Without loss of generality, we assume that $\left|y_{k-1}\right|_{p}>\left|1-\theta y_{k}\right|_{p}^{-1 / 2}$. For $\eta>0$, 
we have

$$
\begin{aligned}
& \sum_{n \in S \cap A_{r}^{+}} \log ^{+} \frac{1}{\left|1-y_{n}\right|_{p}}+\sum_{n \in S \cap A_{r}^{-}} \log ^{+} \frac{1}{\left|1+y_{n}\right|_{p}} \\
= & \log ^{+} \frac{1}{\left|1-\theta y_{k}\right|_{p}}+\log ^{+} \frac{1}{\left|1+\theta y_{k+2}\right|_{p}} \\
= & \eta \log ^{+} \frac{1}{\left|1-\theta y_{k}\right|_{p}}+(1-\eta) \log ^{+} \frac{1}{\left|1-\theta y_{k}\right|_{p}}+\log ^{+} \frac{1}{\left|1+\theta y_{k+2}\right|_{p}} \\
\leq & 2 \eta \log ^{+}\left|y_{k-1}\right|_{p}+\frac{1-\eta}{1-\delta} \log ^{+}\left|y_{k+1}\right|_{p}+\frac{1}{1-\delta} \log ^{+}\left|y_{k+1}\right|_{p} \\
= & 2 \eta \log ^{+}\left|y_{k-1}\right|_{p}+\frac{2-\eta}{1-\delta} \log ^{+}\left|y_{k+1}\right|_{p} .
\end{aligned}
$$

So we can reduce the coefficient of $\log ^{+}\left|y_{k+1}\right|_{p}$ by introducing a contribution from $y_{k-1}$. If $k-1 \in S$, this is not problematic and an upper bound for (19) is

$$
\max \left(\frac{2-\eta}{1-\delta}, 2 \eta\right) \sum_{n \in S} \log ^{+}\left|y_{n}\right|_{p} .
$$

However, if $k-1 \notin S$ then we need to be careful because we will later sum our estimates for (17) over all oscillating sequences. When we do this we might need to "share" the term $k-1$ with another oscillating sequence, in which case it will appear twice in the upper bound and we will need to sum the contributions. Note that the term $k-1$ here cannot be part of a subinterval $I$ of the type considered in case 1 above as such subintervals of oscillating sequences have only \pm 1 points as endpoints. There could, however, be two adjacent oscillating sequences $S_{1}$ and $S_{2}$ both of the type considered in the present case (case 2) that need to share the contribution from $y_{k-1}$. If so, then summing over the contributions for both oscillating sequences would give the upper bound

$$
\frac{2-\eta}{1-\delta} \log ^{+}\left|y_{k-3}\right|_{p}+4 \eta \log ^{+}\left|y_{k-1}\right|_{p}+\frac{2-\eta}{1-\delta} \log ^{+}\left|y_{k+1}\right|_{p}
$$

which, in turn, is bounded from above by

$$
\max \left(\frac{2-\eta}{1-\delta}, 4 \eta\right) \sum_{n=k-3}^{k+1} \log ^{+}\left|y_{n}\right|_{p}
$$


Note that $k-1$ could also be part of an oscillating sequence of the type we are about to consider in case 3 .

Case 3: There is exactly one $k_{1} \in S \cap\left[r_{0}, r\right]$ such that $\left|1-\theta y_{k_{1}}\right|_{p}<\epsilon_{k_{1}}$ for $\theta=-1$ or 1 . Since $S$ has at least two points, we know that either $\left|y_{k_{1}-1}\right|_{p} \geq\left|1-\theta y_{k_{1}}\right|_{p}^{-(1-\delta)}$ or $\left|y_{k_{1}+1}\right|_{p} \geq\left|1-\theta y_{k_{1}}\right|_{p}^{-(1-\delta)}$. Without loss of generality, we assume the latter. Note that since $k_{1} \in S \cap\left[r_{0}, r\right]$, then $k_{1}+1 \in S \cap\left[r_{0}, r+1\right]$. So

$$
\sum_{n \in S \cap A_{r}^{+}} \log ^{+} \frac{1}{\left|1-y_{n}\right|_{p}}+\sum_{n \in S \cap A_{r}^{-}} \log ^{+} \frac{1}{\left|1+y_{n}\right|_{p}}=\log ^{+} \frac{1}{\left|1-\theta y_{k_{1}}\right|_{p}} \leq \frac{1}{1-\delta} \log ^{+}\left|y_{k_{1}+1}\right|_{p} .
$$

It is conceivable that $k_{1}+1$ is adjacent to, or part of, a sequence of the type considered in case 2 in such a way that it plays the role of $k-1$ in the analysis above of that case. In other words, summing over the contributions of these two oscillating sequences in the left side of (10) leads to a term of the form

$$
\left(\frac{1}{1-\delta}+2 \eta\right) \log ^{+}\left|y_{k_{1}+1}\right|_{p}
$$

on the right hand side.

If both $a_{k}-b_{k}-a_{k-2}-b_{k-2}+2 b_{k-1}$ and $a_{k}+b_{k}-a_{k-2}+b_{k-2}-2 b_{k-1}$ are nonzero then combining our results from the above cases, we have

$$
\sum_{n=r_{0}}^{r}\left(\log ^{+} \frac{1}{\left|1-y_{n}\right|_{p}}+\log ^{+} \frac{1}{\left|1+y_{n}\right|_{p}}\right) \leq \tau \sum_{n=r_{0}}^{r+1} \log ^{+}\left|y_{n}\right|_{p}+\Phi_{r}
$$

where

$$
\tau=\max \left(\frac{3}{2(1-\delta)}, \frac{2-\eta}{1-\delta}, 2 \eta, 4 \eta, \frac{1}{1-\delta}+2 \eta\right) .
$$

In particular, choosing $\eta=3 / 8$ and $\delta$ sufficiently small, we have $\tau=3 / 4+$ $(1-\delta)^{-1}<2$. Since $\sum_{p \leq \infty} \Phi_{r}=o\left(h_{r+1}\left(y_{n}\right)\right)$, we see that

$$
h_{r}\left(y_{n}\right) \leq \frac{\tau}{2} h_{r+1}\left(y_{n}\right)+o\left(h_{r+1}\left(y_{n}\right)\right)
$$

so $h_{r}(y)$ grows exponentially with $\mathrm{r}$.

The argument above is based on the fact that Corollary 6 guarantees that if $a_{k}-\theta b_{k}-\theta\left(\theta a_{k-2}+b_{k-2}-2 b_{k-1}\right) \not \equiv 0$ then there can be no special oscillating sequences as defined below. 
Definition 7 The special oscillating sequence $S_{p}$ starting with $k$ is $S_{p}=$ $\{k, k+1, k+2\}$. It is an oscillating sequence of length 3 starting with $k$ in $\mathbb{Z}$ such that $\left|1-\theta y_{k}\right|_{p}<\epsilon_{k},\left|y_{k+1}\right|_{p} \geq \max \left\{\left|1-\theta y_{k}\right|_{p}^{-(1-\delta)},\left|1+\theta y_{k+2}\right|_{p}^{-(1-\delta)}\right\}$ and $\left|1+\theta y_{k+2}\right|_{p}<\epsilon_{k+2}$. Also, we have $\left|y_{k-1}\right|_{p} \leq\left|1-\theta y_{k}\right|_{p}^{-1 / 2}$ and $\left|y_{k+3}\right|_{p} \leq$ $\left|1+\theta y_{k+2}\right|_{p}^{-1 / 2}$.

Note that there are two types of special oscillating sequences depending on whether $\theta=1$ or $\theta=-1$. In order for $h_{r}\left(y_{n}\right)$ to grow sub-exponentially, there must be infinitely many special oscillating sequences. If there are infinitely many special oscillating sequences of both types then both $a_{k}-b_{k}-$ $\left(a_{k-2}+b_{k-2}-2 b_{k-1}\right)$ and $a_{k}+b_{k}+\left(-a_{k-2}+b_{k-2}-2 b_{k-1}\right)$ must vanish, which characterises part (i) of the theorem. The rest of this section will be a careful analysis of the case in which there are infinitely many special oscillating sequences of one kind only, corresponding to a fixed value of $\theta= \pm 1$. For the rest of this section when we refer to special oscillating sequences we mean those sequences of the form $\theta, \infty,-\theta$, for this fixed value of $\theta$ (where " $\infty$ " refers to a large term).

We define $f_{n}$ by

$$
f_{n}=\left(1-\theta y_{n}\right) y_{n+1}-y_{n} .
$$

So $y_{n+1}=\left(f_{n}+y_{n}\right) /\left(1-\theta y_{n}\right), y_{n-1}=\left(y_{n}-f_{n-1}\right) /\left(1+\theta y_{n}\right)$, and (15) yield

$$
y_{n+1}+y_{n-1}=\frac{\left(f_{n}-f_{n-1}\right)+\left(2+\theta f_{n}+\theta f_{n-1}\right) y_{n}}{1-y_{n}^{2}}=\frac{a_{n}+b_{n} y_{n}}{1-y_{n}^{2}} .
$$

Hence

$$
\left(b_{n}-2-\theta f_{n}-\theta f_{n-1}\right) y_{n}=f_{n}-f_{n-1}-a_{n} .
$$

If for all $n, b_{n}-2-\theta f_{n}-\theta f_{n-1}=0$, then $f_{n}-f_{n-1}-a_{n}=0$ and

$$
f_{n}=\frac{1}{2 \theta}\left(\theta a_{n}+b_{n}-2\right) \text {. }
$$

This shows that $y_{n}$ solves the discrete Riccati equation (3).

Next consider the case $b_{n}-2-\theta f_{n}-\theta f_{n-1} \neq 0, \forall n>K$. From (22) we have

$$
y_{n}=\frac{f_{n}-f_{n-1}-a_{n}}{b_{n}-2-\theta f_{n}-\theta f_{n-1}} .
$$


Taking the logarithmic height of both sides of (23) and using some elementary properties of heights, we have

$$
\begin{aligned}
h\left(y_{n}\right) & =h\left(\frac{f_{n}-f_{n-1}-a_{n}}{b_{n}-2-\theta f_{n}-\theta f_{n-1}}\right) \\
& \leq h\left(f_{n}-f_{n-1}-a_{n}\right)+h\left(\frac{1}{b_{n}-2-\theta f_{n}-\theta f_{n-1}}\right) \\
& =h\left(f_{n}-f_{n-1}-a_{n}\right)+h\left(b_{n}-2-\theta f_{n}-\theta f_{n-1}\right) \\
& \leq 2 h\left(f_{n}\right)+2 h\left(f_{n-1}\right)+h\left(a_{n}\right)+h\left(b_{n}\right)+\log 24 .
\end{aligned}
$$

Summing both sides of the inequality above and using the fact that $h_{r}\left(f_{n}\right)$ is a non-decreasing function of $n$, we have

$$
h_{r}\left(y_{n}\right) \leq 4 h_{r+1}\left(f_{n}\right)+h_{r}\left(a_{n}\right)+h_{r}\left(b_{n}\right)+\left(r-r_{0}+1\right) \log 24 .
$$

From (21) we have

$$
f_{n}+\theta=\theta\left(1-\theta y_{n}\right)\left(1+\theta y_{n+1}\right) .
$$

For every prime $p \leq \infty$, we define a set $C_{p} \subset \mathbb{Z}$ such that it consists of all the big terms in special oscillating sequences i.e. the terms $\infty$ s in the form: $\theta, \infty,-\theta$. For a fixed prime $p$ and sufficiently large $r_{0}$, we have

$$
\begin{aligned}
& \sum_{n=r_{0}}^{r} \log ^{+} \frac{1}{\left|f_{n}+\theta\right|_{p}}=\sum_{\substack{n=r_{0} \\
n \in C_{p}}}^{r} \log ^{+} \frac{1}{\left|f_{n}+\theta\right|_{p}}+\sum_{\substack{n=r_{0} \\
n+1 \in C_{p}}}^{r} \log ^{+} \frac{1}{\left|f_{n}+\theta\right|_{p}} \\
& \sum_{\substack{n=r_{0} \\
n \notin C_{p} \text { and } n+1 \notin C_{p}}}^{r} \log ^{+} \frac{1}{\left|f_{n}+\theta\right|_{p}} .
\end{aligned}
$$

In the above inequality we split the interval $\left[r_{0}, r\right]$ into points that are in special oscillating sequences (where $n, n+1 \in C_{p}$ ) and points in any other oscillating sequence that is not special. Note that for $n \in C_{p}$, we have $\left|1+\theta y_{n+1}\right|_{p}^{-(1-\delta)} \leq\left|y_{n}\right|_{p}$. Therefore, for $n \in C_{p}$ we have

$$
\begin{aligned}
\log ^{+} \frac{1}{\left|f_{n}+\theta\right|_{p}} & =\log ^{+} \frac{1}{\left|1-\theta y_{n}\right|_{p} \cdot\left|1+\theta y_{n+1}\right|_{p}} \leq \log ^{+}\left|1-\theta y_{n}\right|_{p}^{-1} \cdot\left|y_{n}\right|_{p}^{\frac{1}{1-\delta}} \\
& =\log ^{+}\left|1-\theta y_{n}\right|_{p}^{-1} \cdot\left|y_{n}\right|_{p}^{\frac{\delta+1-\delta}{1-\delta}}=\log ^{+}\left|1-\theta y_{n}\right|_{p}^{-1} \cdot\left|y_{n}\right|_{p} \cdot\left|y_{n}\right|_{p}^{\frac{\delta}{1-\delta}} \\
& \leq \frac{\delta}{1-\delta} \log ^{+}\left|y_{n}\right|_{p}+\log ^{+}\left|\frac{y_{n}}{1-\theta y_{n}}\right|_{p} .
\end{aligned}
$$


Since $\left|y_{n}\right|_{p}$ is big, it is away from $\theta$ and $-\theta$. If $p<\infty$, then $\left|y_{n}\right|_{p}=\mid \theta-$ $\left.\theta\left(1-\theta y_{n}\right)\right|_{p} \leq \max \left\{1,\left|1-\theta y_{n}\right|_{p}\right\}=\left|1-\theta y_{n}\right|_{p}$, since $\left|y_{n}\right|_{p}>1$. Hence, the term $\log ^{+}\left|\frac{y_{n}}{1-\theta y_{n}}\right|_{p}$ vanishes. For $p=\infty$ we have the following relation $\epsilon_{n+1}^{-\delta}<\epsilon_{n+1}^{-(1-\delta)}<\left|1+\theta y_{n+1}\right|_{\infty}^{-(1-\delta)} \leq\left|y_{n}\right|_{\infty} \leq 1+\left|1-\theta y_{n}\right|_{\infty}$ which yields $\epsilon_{n+1}^{-\delta}-1 \leq\left|1-\theta y_{n}\right|_{\infty}$. Consequently, $\frac{1}{\left|1-\theta y_{n}\right|_{\infty}} \leq \frac{1}{\epsilon_{n+1}^{-\delta}-1}$. Starting with $\left|y_{n}\right|_{\infty} \leq 1+\left|1-\theta y_{n}\right|_{\infty}$ then dividing both sides by $\left|1-\theta y_{n}\right|_{\infty}$ implies $\frac{\left|y_{n}\right|_{\infty}}{\left|1-\theta y_{n}\right|_{\infty}} \leq$ $\frac{1}{\left|1-\theta y_{n}\right|_{\infty}}+1 \leq \frac{1}{\epsilon_{n+1}^{-\delta}-1}+1$. Therefore, $\left|\frac{y_{n}}{1-\theta y_{n}}\right|_{\infty} \leq \frac{1}{4}+1=\frac{5}{4}$ since $5 \leq \epsilon_{n+1}^{-\delta}$, giving

$$
\sum_{p \leq \infty} \sum_{\substack{n=r_{0} \\ n \in C_{p}}}^{r} \log ^{+} \frac{1}{\left|f_{n}+\theta\right|_{p}} \leq \frac{\delta}{1-\delta} h_{r}\left(y_{n}\right)+\left(r-r_{0}+1\right) \log (5 / 4) .
$$

Similarly,

$$
\sum_{p \leq \infty} \sum_{\substack{n=r_{0} \\ n+1 \in C_{p}}}^{r} \log ^{+} \frac{1}{\left|f_{n}+\theta\right|_{p}} \leq \frac{\delta}{1-\delta} h_{r+1}\left(y_{n}\right)+\left(r-r_{0}+1\right) \log (5 / 4)
$$

Summing over all $p \leq \infty$ in (26) and using (25), (27) and (28) yields

$$
\begin{array}{r}
h_{r}\left(f_{n}\right)-\left(r-r_{0}+1\right) \log 2 \leq h_{r}\left(\frac{1}{f_{n}+\theta}\right) \leq \frac{2 \delta}{1-\delta} h_{r+1}\left(y_{n}\right)+2\left(r-r_{0}+1\right) \log (5 / 4) \\
+\sum_{p \leq \infty}\left\{\sum_{\substack{n=r_{0} \\
n+1 \notin C_{p}}}^{r} \log ^{+} \frac{1}{\left|1-\theta y_{n}\right|_{p}}+\sum_{\substack{n=r_{0} \\
n \notin C_{p}}}^{r} \log ^{+} \frac{1}{\left|1+\theta y_{n+1}\right|_{p}}\right\} .
\end{array}
$$

Therefore,

$$
h_{r}\left(f_{n}\right) \leq \frac{2 \delta}{1-\delta} h_{r+1}\left(y_{n}\right)+\left(r-r_{0}+1\right) \log (25 / 8)+B_{r+1},
$$

where

$$
B_{r}=\sum_{p \leq \infty}\left\{\sum_{\substack{n=r_{0} \\ n+1 \notin C_{p}}}^{r} \log ^{+} \frac{1}{\left|1-\theta y_{n}\right|_{p}}+\sum_{\substack{n=r_{0} \\ n-1 \notin C_{p}}}^{r} \log ^{+} \frac{1}{\left|1+\theta y_{n}\right|_{p}}\right\}
$$


From our previous analysis of oscillating sequences that are not special, it follows from (20) that

$$
B_{r} \leq \tau \sum_{p \leq \infty} \sum_{\substack{n=r_{0} \\ n \notin C_{p}}}^{r+1} \log ^{+}\left|y_{n}\right|_{p}+R_{r}
$$

Recall that $\tau<2$ and $R_{r}$ is an expression that involves the summed logarithmic heights of the coefficients $a_{n}$ and $b_{n}$. Applying the shift $r \rightarrow r+1$ in (29) and (31), then using the result in (24) yields

$$
h_{r}\left(y_{n}\right) \leq \frac{8 \delta}{1-\delta} h_{r+2}\left(y_{n}\right)+4 \tau \sum_{p \leq \infty} \sum_{\substack{n=r_{0} \\ n \notin C_{p}}}^{r+3} \log ^{+}\left|y_{n}\right|_{p}+\widehat{R}_{r+2},
$$

where $\widehat{R}_{r+2}=o\left(h_{r+2}\left(y_{n}\right)\right)$.

Now we consider the following inequality

$$
\begin{aligned}
& \sum_{p \leq \infty} \sum_{n=r_{0}}^{r}\left\{\log ^{+} \frac{1}{\left|1-y_{n}\right|_{p}}+\log ^{+} \frac{1}{\left|1+y_{n}\right|_{p}}\right\} \\
\leq & \sum_{p \leq \infty}\left\{\sum_{\substack{n=r_{0} \\
n+1 \in C_{p}}}^{r} \log ^{+} \frac{1}{\left|1-\theta y_{n}\right|_{p}}+\sum_{\substack{n=r_{0} \\
n-1 \in C_{p}}}^{r} \log ^{+} \frac{1}{\left|1+\theta y_{n}\right|_{p}}\right\}+B_{r} .
\end{aligned}
$$


Recall that if $n+1 \in C_{p}$ (or $n-1 \in C_{p}$ ), then $\left|y_{n+1}\right|_{p} \geq\left|1-\theta y_{n}\right|_{p}^{-(1-\delta)}$ (or $\left.\left|y_{n-1}\right|_{p} \geq\left|1+\theta y_{n}\right|_{p}^{-(1-\delta)}\right)$. Using this fact and (31) we have

$$
\begin{aligned}
& \sum_{p \leq \infty} \sum_{n=r_{0}}^{r}\left\{\log ^{+} \frac{1}{\left|1-y_{n}\right|_{p}}+\log ^{+} \frac{1}{\left|1+y_{n}\right|_{p}}\right\} \\
& \leq \frac{2}{1-\delta} \sum_{p \leq \infty} \sum_{\substack{n=r_{0} \\
n \in C_{p}}}^{r+1} \log ^{+}\left|y_{n}\right|_{p}+\tau \sum_{p \leq \infty} \sum_{\substack{n=r_{0} \\
n \notin C_{p}}}^{r+1} \log ^{+}\left|y_{n}\right|_{p}+R_{r} \\
& =\frac{2}{1-\delta} \sum_{p \leq \infty} \sum_{\substack{n=r_{0} \\
n \in C_{p}}}^{r+1} \log ^{+}\left|y_{n}\right|_{p}+\frac{2}{1-\delta} \sum_{p \leq \infty} \sum_{\substack{n=r_{0} \\
n \notin C_{p}}}^{r+1} \log ^{+}\left|y_{n}\right|_{p} \\
& -\frac{2}{1-\delta} \sum_{p \leq \infty} \sum_{\substack{n=r_{0} \\
n \notin C_{p}}}^{r+1} \log ^{+}\left|y_{n}\right|_{p}+\tau \sum_{p \leq \infty} \sum_{\substack{n=r_{0} \\
n \notin C_{p}}}^{r+1} \log ^{+}\left|y_{n}\right|_{p}+R_{r} \\
& =\frac{2}{1-\delta} h_{r+1}\left(y_{n}\right)-\left(\frac{2}{1-\delta}-\tau\right) \sum_{p \leq \infty} \sum_{\substack{n=r_{0} \\
n \notin C_{p}}}^{r+1} \log ^{+}\left|y_{n}\right|_{p}+R_{r} .
\end{aligned}
$$

This implies that

$$
2 h_{r}\left(y_{n}\right) \leq \frac{2}{1-\delta} h_{r+1}\left(y_{n}\right)-\left(\frac{2}{1-\delta}-\tau\right) \sum_{p \leq \infty} \sum_{\substack{n=r_{0} \\ n \notin C_{p}}}^{r+1} \log ^{+}\left|y_{n}\right|_{p}+\widetilde{R_{r}}
$$

where $\widetilde{R_{r}}=o\left(h_{r}\left(y_{n}\right)\right)$ as $r \rightarrow \infty$.

Considering the two inequalities in (32) and (34), we have two cases to consider depending on whether the expression $\sum_{p \leq \infty} \sum_{\substack{n=r_{0} \\ n \notin C_{p}}}^{r+1} \log ^{+}\left|y_{n}\right|_{p}$ is very small compared to $h_{r+1}\left(y_{n}\right)$ on a large set. In either case we obtain an inequality of the form $h_{r+s}\left(y_{n}\right) \geq \alpha h_{r}\left(y_{n}\right)$, for some $\alpha<1$ and $s>0$, on a set of infinite logarithmic measure, which implies conclusion (iii) of the theorem.

Case 1: Assume that there is a sufficiently small constant $c>0$ such that

$$
\sum_{p \leq \infty} \sum_{\substack{n=r_{0} \\ n \notin C_{p}}}^{r+1} \log ^{+}\left|y_{n}\right|_{p} \leq c h_{r+1}\left(y_{n}\right)
$$


on a set of infinite discrete logarithmic measure. Then (32) implies

$$
h_{r}\left(y_{n}\right) \leq\left(\frac{8 \delta}{1-\delta}+4 \tau c\right) h_{r+3}\left(y_{n}\right)+\widehat{R}_{r+2},
$$

on a set of infinite discrete logarithmic measure.

Case 2: Assume that

$$
\sum_{p \leq \infty} \sum_{\substack{n=r_{0} \\ n \notin C_{p}}}^{r+1} \log ^{+}\left|y_{n}\right|_{p}>c h_{r+1}\left(y_{n}\right)
$$

on a set of infinite discrete logarithmic measure. Using this inequality in (34) yields

$$
2 h_{r}\left(y_{n}\right) \leq\left[\frac{2}{1-\delta}-\left(\frac{2}{1-\delta}-\tau\right) c\right] h_{r+1}\left(y_{n}\right)+\widetilde{R}_{r}
$$

Since $\left[\frac{2}{1-\delta}-\left(\frac{2}{1-\delta}-\tau\right) c\right]<2$ for sufficiently small $\delta$.

Conclusion (iii) of the theorem follows from the following with $w_{r}=$ $h_{r}\left(y_{n}\right)$.

Lemma 8 Let $\left(w_{n}\right)_{n \geq n_{0}}\left(n_{0}>0\right)$ be a non-decreasing sequence of positive numbers. For a fixed real number $\alpha>1$ and a fixed positive integer $s$ we define

$$
F=\left\{n \geq n_{0}: w_{n+s} \geq \alpha w_{n}\right\}
$$

If $F$ has infinite discrete logarithmic measure, i.e. $\sum_{n \in F} \frac{1}{n}=\infty$, then

$$
\limsup _{r \rightarrow \infty} \frac{\log \log w_{r}}{\log r} \geq 1
$$

Proof: Define a sequence $\left(r_{n}\right)$ using induction as follows. Let $r_{0}=\min (F)$ and for all $n>0$, define $r_{n}=\min \left(F \cap\left[r_{n-1}+s, \infty\right)\right)$. Hence, $r_{n+1} \geq r_{n}+s$ and $F \subseteq \cup_{n=0}^{\infty}\left[r_{n}, r_{n}+s\right]$. This yields $w_{r_{n+1}} \geq w_{r_{n}+s} \geq \alpha w_{r_{n}}$ for all $n \geq 0$. Iterating this relation recursively yields

$$
w_{r_{n}} \geq \alpha^{n} w_{r_{0}} .
$$


We use the notation $\lfloor x\rfloor$ to denote the integer part of $x$ in the following chain of inequalities. Assume that there is a constant $\varepsilon>0$ and an integer $m>1$ such that $r_{n} \geq n^{1+\varepsilon}$ for all $n>m$. Then there is a constant $E$ such that

$$
\begin{aligned}
\sum_{j \in F} \frac{1}{j} & \leq E+\sum_{n=m}^{\infty} \sum_{k=\left\lfloor n^{1+\varepsilon}\right\rfloor}^{\left\lfloor n^{1+\varepsilon}\right\rfloor+s} \frac{1}{k} \leq E+\sum_{n=m}^{\infty} \int_{n^{1+\varepsilon}-2}^{n^{1+\varepsilon}+s} \frac{\mathrm{d} t}{t} \\
& \leq E+\sum_{n=m}^{\infty}\left((s+2) n^{-(1+\varepsilon)}+O\left(n^{-2(1+\varepsilon)}\right)\right)<\infty
\end{aligned}
$$

But this is a contradiction to our assumption that $F$ has infinite discrete logarithmic measure. Therefore, there exists a subsequence $\left(r_{n_{k}}\right)$ such that $r_{n_{k}}<n_{k}^{1+\varepsilon}$ for all $k \geq 0$. From (37) we have $w_{r_{n_{k}}} \geq \alpha^{n_{k}} w_{r_{0}}$. Hence,

$$
\begin{aligned}
\limsup _{r \rightarrow \infty} \frac{\log \log w_{r}}{\log r} & \geq \limsup _{k \longrightarrow \infty} \frac{\log \log w_{r_{n_{k}}}}{\log r_{n_{k}}} \geq \limsup _{k \longrightarrow \infty} \frac{\log \log \alpha^{n_{k}} w_{r_{0}}}{\log n_{k}^{1+\varepsilon}} \\
& =\limsup _{k \longrightarrow \infty} \frac{\log \left(n_{k} \log \alpha+\log w_{r_{0}}\right)}{(1+\varepsilon) \log n_{k}} \geq \frac{1}{1+\varepsilon} .
\end{aligned}
$$

Since $\varepsilon>0$ is arbitrary small number, this proves the lemma.

This concludes the case in which $c_{n} \equiv 0$. The cases $c_{n} \equiv \pm 2$ are similar except that the existence of Riccati solutions is prohibited by the assumption in the theorem that the degree of the right side of equation (11) is two.

\section{Proof of Theorem 2}

For a fixed absolute value $|\cdot|_{p}$, assume that $\left|1-\theta y_{k}\right|_{p}<\epsilon_{k}$ for some $k>K$, where $\theta=-1$ or 1 . First we rewrite equation (15) as

$$
y_{k+1}+y_{k-1}=\frac{1 / 2\left(a_{k}+\theta b_{k}\right)}{1-\theta y_{k}}+\frac{1 / 2\left(a_{k}-\theta b_{k}\right)}{1+\theta y_{k}} .
$$

It follows that

$$
A_{k}:=y_{k+1}-\frac{1 / 2\left(a_{k}+\theta b_{k}\right)}{1-\theta y_{k}}=\frac{1 / 2\left(a_{k}-\theta b_{k}\right)}{1+\theta y_{k}}-y_{k-1} .
$$

We begin by considering non-Archimedean absolute values $(p<\infty)$. In this case

$$
\left|A_{k}\right|_{p} \leq \max \left\{\frac{|1 / 2|_{p} \cdot\left|a_{k}-\theta b_{k}\right|_{p}}{\left|1+\theta y_{k}\right|_{p}},\left|y_{k-1}\right|_{p}\right\} .
$$


Note that $\epsilon_{k}^{\delta} \leq|2|_{p} \leq \max \left\{\left|1-\theta y_{k}\right|_{p},\left|1+\theta y_{k}\right|_{p}\right\}$. If $\left|1-\theta y_{k}\right|_{p}>\left|1+\theta y_{k}\right|_{p}$, then we obtain the contradiction $\epsilon_{k}^{\delta}<\epsilon$. Hence $\epsilon_{k}^{\delta} \leq \max \left\{\left|1-\theta y_{k}\right|_{p}, \mid 1+\right.$ $\left.\left.\theta y_{k}\right|_{p}\right\}=\left|1+\theta y_{k}\right|_{p}$. This implies $\left|1+\theta y_{k}\right|_{p}{ }^{-1} \leq \epsilon_{k}^{-\delta}<\left|1-\theta y_{k}\right|_{p}^{-\delta}$. Using this relation and (16) in (40) yields $\left|A_{k}\right|_{p} \leq \max \left\{\left|1-\theta y_{k}\right|_{p}^{-2 \delta},\left|1-\theta y_{k}\right|_{p}^{-1 / 2}\right\}=$ $\left|1-\theta y_{k}\right|_{p}^{-1 / 2}$.

Consider

$$
B_{k}:=\left(y_{k+2}+\theta\right)-\left(\theta-\frac{2 b_{k+1}}{a_{k}+\theta b_{k}}\right)\left(1-\theta y_{k}\right)
$$

Incrementing equation (15) we obtain

$$
\begin{aligned}
B_{k}=\frac{a_{k+1}}{\left(1-y_{k+1}\right)\left(1+y_{k+1}\right)} & +\frac{b_{k+1}}{\left(1-y_{k+1}\right)\left(1+y_{k+1}\right)\left(y_{k+1}-A_{k}\right)} \\
& -\frac{b_{k+1} A_{k} y_{k+1}}{\left(1-y_{k+1}\right)\left(1+y_{k+1}\right)\left(y_{k+1}-A_{k}\right)} .
\end{aligned}
$$

Now

$$
\left|1-\theta y_{k}\right|_{p}^{-(1-\delta)}<\frac{\epsilon_{k}^{\delta}}{\left|1-\theta y_{k}\right|_{p}} \leq \frac{|1 / 2|_{p} \cdot\left|a_{k}+\theta b_{k}\right|_{p}}{\left|1-\theta y_{k}\right|_{p}}=\left|y_{k+1}-A_{k}\right|_{p} .
$$

So $\left|1-\theta y_{k}\right|_{p}^{-(1-\delta)}<\max \left\{\left|y_{k+1}\right|_{p},\left|1-\theta y_{k}\right|_{p}^{-1 / 2}\right\} \leq \max \left\{\left|y_{k+1}\right|_{p},\left|1-\theta y_{k}\right|_{p}^{-(1-\delta)}\right\}$ $=\left|y_{k+1}\right|_{p}$. Hence, $\epsilon_{k}^{-(1-\delta)}<\left|1-\theta y_{k}\right|_{p}^{-(1-\delta)} \leq\left|y_{k+1}\right|_{p}=\left|1-\left(1 \pm y_{k+1}\right)\right|_{p} \leq$ $\max \left\{1,\left|1 \pm y_{k+1}\right|_{p}\right\} \leq \max \left\{\epsilon^{-(1-\delta)},\left|1 \pm y_{k+1}\right|_{p}\right\}$, giving

$$
\left|1 \pm y_{k+1}\right|_{p}^{-1} \leq\left|1-\theta y_{k}\right|_{p}^{1-\delta} \text {. }
$$

Moreover, we have from the first part of the theorem that

$$
\left|y_{k+1}\right|_{p} \leq \max \left\{\frac{|1 / 2|_{p} \cdot\left|a_{k}+\theta b_{k}\right|_{p}}{\left|1-\theta y_{k}\right|_{p}},\left|A_{k}\right|_{p}\right\} \leq\left|1-\theta y_{k}\right|_{p}^{-(1+\delta)} .
$$

Taking the $p$-adic absolute value of equation (42) and using the estimates above, we get

$$
\left|B_{k}\right|_{p} \leq \max \left\{\left|1-\theta y_{k}\right|_{p}^{2-3 \delta},\left|1-\theta y_{k}\right|_{p}^{3-4 \delta},\left|1-\theta y_{k}\right|_{p}^{3 / 2-5 \delta}\right\}
$$

Hence $\left|B_{k}\right|_{p} \leq\left|1-\theta y_{k}\right|_{p}^{3 / 2-5 \delta}$, as required. 
Next we have

$$
C_{k}:=y_{k+3}-\frac{\left(a_{k+2}-\theta b_{k+2}-\theta\left(\theta a_{k}+b_{k}-2 b_{k+1}\right)\right)}{2\left(1+\theta y_{k+2}\right)} .
$$

Incrementing equation (15) twice and eliminating $y_{k+3}$ from the above yields

$$
C_{k}=\frac{1 / 2\left(a_{k+2}+\theta b_{k+2}\right)}{1-\theta y_{k+2}}-\frac{a_{k}+\theta b_{k}}{2\left(1-\theta y_{k}\right)}+\frac{\theta\left(\theta a_{k}+b_{k}-2 b_{k+1}\right)}{2\left(1+\theta y_{k+2}\right)}-A_{k} .
$$

Combining the two middle terms and using part (ii) in the numerator gives

$$
C_{k}=\frac{1 / 2\left(a_{k+2}+\theta b_{k+2}\right)}{1-\theta y_{k+2}}-\frac{B_{k}\left(a_{k}+\theta b_{k}\right)}{2 \theta\left(1+\theta y_{k+2}\right)\left(1-\theta y_{k}\right)}-A_{k} .
$$

From part (ii) of the theorem, we have

$$
\begin{aligned}
\left|1+\theta y_{k+2}\right|_{p} & \leq \max \left\{\frac{\left|\theta a_{k}+b_{k}-2 b_{k+1}\right|_{p}}{\left|a_{k}+\theta b_{k}\right|_{p}}\left|1-\theta y_{k}\right|_{p},\left|B_{k}\right|_{p}\right\} \\
& \leq \max \left\{\left|1-\theta y_{k}\right|_{p}^{1-2 \delta},\left|1-\theta y_{k}\right|_{p}^{3 / 2-5 \delta}\right\}<\epsilon_{k}^{1-2 \delta},
\end{aligned}
$$

where we have used (16). Also, $\epsilon_{k}^{\delta} \leq|2|_{p} \leq \max \left\{\left|1+\theta y_{k+2}\right|_{p},\left|1-\theta y_{k+2}\right|_{p}\right\} \leq$ $\max \left\{\epsilon_{k}^{1-2 \delta},\left|1-\theta y_{k+2}\right|_{p}\right\}=\left|1-\theta y_{k+2}\right|_{p}$. Hence

$$
\left|1-\theta y_{k+2}\right|_{p}^{-1} \leq \epsilon_{k}^{-\delta}<\left|1-\theta y_{k}\right|_{p}^{-\delta} .
$$

Note that if $\left|\theta a_{k}+b_{k}-2 b_{k+1}\right|_{p} \not \equiv 0$, then

$$
\begin{gathered}
\left|1-\theta y_{k}\right|_{p}^{1+2 \delta}=\left|1-\theta y_{k}\right|_{p} \cdot\left|1-\theta y_{k}\right|_{p}^{2 \delta}<\left|1-\theta y_{k}\right|_{p} \epsilon_{k}^{2 \delta} \\
\leq \frac{\left|\theta a_{k}+b_{k}-2 b_{k+1}\right|_{p}}{\left|a_{k}+\theta b_{k}\right|_{p}}\left|1-\theta y_{k}\right|_{p}=\left|\left(1+\theta y_{k+2}\right)-B_{k}\right|_{p} \leq \max \left\{\left|1+\theta y_{k+2}\right|_{p},\left|B_{k}\right|_{p}\right\} \\
\leq \max \left\{\left|1+\theta y_{k+2}\right|_{p},\left|1-\theta y_{k}\right|_{p}^{3 / 2-5 \delta}\right\}=\left|1+\theta y_{k+2}\right|_{p} .
\end{gathered}
$$

So $\left|1+\theta y_{k+2}\right|_{p}^{-1}<\left|1-\theta y_{k}\right|_{p}^{-(1+2 \delta)}$.

If $\left|\theta a_{k}+b_{k}-2 b_{k+1}\right|_{p} \not \equiv 0$ then the second term in (46) satisfies

$$
\left|\frac{B_{k}\left(a_{k}+\theta b_{k}\right)}{2 \theta\left(1+\theta y_{k+2}\right)\left(1-\theta y_{k}\right)}\right|_{p} \leq\left|1-\theta y_{k}\right|_{p}^{1 / 2-6 \delta} \cdot\left|1+\theta y_{k+2}\right|_{p}^{-1} \leq\left|1-\theta y_{k}\right|_{p}^{-1 / 2-8 \delta},
$$


where we have used (16). From equation (46) we have

$$
\begin{gathered}
\left|C_{k}\right|_{p} \leq \max \left\{\frac{|1 / 2|_{p} \cdot\left|a_{k+2}+\theta b_{k+2}\right|_{p}}{\left|1-\theta y_{k+2}\right|_{p}},\left|\frac{B_{k}\left(a_{k}+\theta b_{k}\right)}{2 \theta\left(1+\theta y_{k+2}\right)\left(1-\theta y_{k}\right)}\right|_{p},\left|A_{k}\right|_{p}\right\} \\
\leq \max \left\{\left|1-\theta y_{k}\right|_{p}^{-2 \delta},\left|1-\theta y_{k}\right|_{p}^{-1 / 2-8 \delta},\left|1-\theta y_{k}\right|_{p}^{-1 / 2}\right\}=\left|1-\theta y_{k}\right|_{p}^{-1 / 2-8 \delta},
\end{gathered}
$$

where we have used (48), (49) and the first part of the theorem. From (47) we have $\left|1+\theta y_{k+2}\right|_{p} \leq\left|1-\theta y_{k}\right|_{p}^{1-2 \delta}$. So for sufficiently small $\delta$

$$
\left|C_{k}\right|_{p} \leq\left|1-\theta y_{k}\right|_{p}^{-(1 / 2+8 \delta)} \leq\left|1+\theta y_{k+2}\right|_{p}^{-1 / 2-10 \delta} \leq\left|1+\theta y_{k+2}\right|_{p}^{-2 / 3-2 \delta} .
$$

Now if $\left|\theta a_{k}+b_{k}-2 b_{k+1}\right|_{p} \equiv 0$, then the upper bound on the second term in (46) is

$$
\left|\frac{B_{k}\left(a_{k}+\theta b_{k}\right)}{2 \theta\left(1+\theta y_{k+2}\right)\left(1-\theta y_{k}\right)}\right|_{p}=\frac{\left|1+\theta y_{k+2}\right|_{p} \cdot\left|a_{k}+\theta b_{k}\right|_{p}}{|2|_{p} \cdot\left|1+\theta y_{k+2}\right|_{p} \cdot\left|1-\theta y_{k}\right|_{p}} \leq\left|1-\theta y_{k}\right|_{p}^{-(1+\delta)} .
$$

Consequently,

$$
\begin{aligned}
\left|C_{k}\right|_{p} & \leq \max \left\{\frac{|1 / 2|_{p} \cdot\left|a_{k+2}+\theta b_{k+2}\right|_{p}}{\left|1-\theta y_{k+2}\right|_{p}},\left|\frac{B_{k}\left(a_{k}+\theta b_{k}\right)}{2 \theta\left(1+\theta y_{k+2}\right)\left(1-\theta y_{k}\right)}\right|_{p},\left|A_{k}\right|_{p}\right\} \\
& \leq \max \left\{\left|1-\theta y_{k}\right|_{p}^{-2 \delta},\left|1-\theta y_{k}\right|_{p}^{-(1+\delta)},\left|1-\theta y_{k}\right|_{p}^{-1 / 2}\right\}=\left|1-\theta y_{k}\right|_{p}^{-(1+\delta)} .
\end{aligned}
$$

Since $\left|1+\theta y_{k+2}\right|_{p}=\left|B_{k}\right|_{p} \leq\left|1-\theta y_{k}\right|_{p}^{3 / 2-5 \delta}$, it yields that $\left|1-\theta y_{k}\right|_{p}^{-(1+\delta)} \leq$ $\left|1+\theta y_{k+2}\right|_{p}^{\frac{-(1+\delta)}{3 / 2-5 \delta}} \leq\left|1+\theta y_{k+2}\right|_{p}^{-2 / 3-2 \delta}$. Hence, $\left|C_{k}\right|_{p} \leq\left|1+\theta y_{k+2}\right|_{p}^{-2 / 3-2 \delta}$ which proves the last part of the theorem for the non-Archimedean absolute value.

Estimates for the Archimedean case $(p=\infty)$ are similar to the above. Here we will derive the estimate for $\left|A_{k}\right|_{p}$ only. Since $2=|2|_{\infty} \leq\left|1-\theta y_{k}\right|_{\infty}+$ $\left|1+\theta y_{k}\right|_{\infty}<\epsilon_{k}+\left|1+\theta y_{k}\right|_{\infty}<1+\left|1+\theta y_{k}\right|_{\infty}$, we have $\left|1+\theta y_{k}\right|_{\infty}^{-1}<1$. So equation (39) gives

$$
\begin{aligned}
\left|A_{k}\right|_{\infty} & \leq \frac{|1 / 2|_{\infty} \cdot\left|a_{k}-\theta b_{k}\right|_{\infty}}{\left|1+\theta y_{k}\right|_{\infty}}+\left|y_{k-1}\right|_{\infty} \leq \frac{1}{10} \epsilon_{k}^{-\delta} \cdot 1+\left|1-\theta y_{k}\right|_{\infty}^{-1 / 2} \\
& \leq \frac{1}{10}\left|1-\theta y_{k}\right|_{\infty}^{-\delta}+\left|1-\theta y_{k}\right|_{\infty}^{-1 / 2} \leq \frac{11}{10}\left|1-\theta y_{k}\right|_{\infty}^{-1 / 2},
\end{aligned}
$$

for sufficiently small $\delta$, which proves the first part of the theorem for $p=\infty$. 


\section{Acknowledgements}

The first author thanks Sultan Qaboos University for supporting her through grant IG/SCI/DOMAS/12/01. The second author gratefully acknowledges support from EPSRC grants EP/C54319X/2 and EP/I013334/1.

\section{References}

[1] Abarenkova N, Anglès d'Auriac J-Ch, Boukraa S, Hassani S, and Maillard J-M 1999 Topological entropy and Arnold complexity for twodimensional mappings. Phys. Lett. A 262 44-49.

[2] Ablowitz M J, Halburd R and Herbst B 2000 On the extension of the Painlevé property to difference equations. Nonlinearity 13 889-905

[3] Al-Ghassani A 2010 Measures of growth of discrete rational equations, $\mathrm{PhD}$ thesis, Loughborough University

[4] Anglès d'Auriac J-C, Maillard J-M, and Viallet C-M 2006 On the complexity of some birational transformations. J.Phys. A 39 3641-3654.

[5] Bellon M and Viallet C-M 1999 Algebraic Entropy. Comm. Math. Phys. $204425-437$

[6] Falqui G and Viallet C-M 1993 Singularity, complexity, and quasiintegrability of rational mappings. Comm. Math. Phys. 154 111-125

[7] Fordy A P and Marsh R 2011 Cluster mutation-periodic quivers and associated Laurent sequences. J. Algebraic Combin. 34 19-66

[8] Grammaticos B, Ramani A and Papageorgiou V 1991 Do integrable mappings have the Painlevé property? Phys. Rev. Lett. 67 1825-1827

[9] Grammaticos B, Halburd R G, Ramani A and Viallet C-M 2009 How to detect the integrability of discrete systems. J. Phys. A 42454002

[10] Halburd R G 2005 Diophantine integrability. J. Phys. A: Math. Gen. 38 L263-L269

[11] Halburd R G and Korhonen R J 2007 Finite-order meromorphic solutions and the discrete Painlevé equations. Proc. London Math. Soc. $\mathbf{9 4}$ 443-474 
[12] Halburd R G and Korhonen R J 2007 Meromorphic solutions of difference equations, integrability and the discrete Painlevé equations. $J$. Phys. A. 40 R1-R38

[13] Halburd R G and Morgan W 2013 Diophantine integrability and a discrete Painlevé equation. To appear

[14] Hietarinta J and Viallet C-M, 1998 Singularity confinement and chaos in discrete systems. Phys. Rev. Lett. 81 325-328

[15] Hone A N W 2006 Diophantine non-integrability of a third-order recurrence with the Laurent property. J. Phys. A 39 L171-L177

[16] Hone A N W and Petrera M 2009 Three-dimensional discrete systems of Hirota-Kimura type and deformed Lie-Poisson algebras. J. Geom. Mech. 1 (2009) 55-85

[17] Hone A N W and Swart C 2008 Integrality and the Laurent phenomenon for Somos 4 and Somos 5 sequences. Math. Proc. Cambridge Philos. Soc. $14565-85$

[18] Nijhoff F W and Papageorgiou V 1991 Similarity reductions of integrable lattices and discrete analogues of the Painlevé II equation. Phys. Lett. A 153 337-344

[19] Ramani A, Grammaticos B and Hietarinta J 1991 Discrete versions of the Painlevé equations. Phys. Rev. Lett. 67 1829-1832

[20] Veselov A P 1992 Growth and Integrability in the Dynamics of Mappings. Comm. Math. Phys. 145 181-193

[21] Vojta P 1987 Diophantine Approximations and Value Distribution Theory (Lecture Notes in Math. vol 1239, Berlin: Springer-Verlag) 